(C) The Authors 2015. This is an Open Access article, distributed under the terms of the Creative

Commons Attribution licence (http://creativecommons.org/licenses/by/4.0/), which permits unrestricted

re-use, distribution, and reproduction in any medium, provided the original work is properly cited.

\title{
Cross-sectional association between serum concentrations of $n-3$ long-chain PUFA and depressive symptoms: results in Japanese community dwellers
}

\author{
Chika Horikawa $^{1,2 *}$, Rei Otsuka ${ }^{1}$, Yuki Kato ${ }^{1,3}$, Yukiko Nishita $^{1}$, Chikako Tange ${ }^{1}$, Saki Kakutani ${ }^{2}$, \\ Tomohiro Rogi ${ }^{2}$, Hiroshi Kawashima ${ }^{2}$, Hiroshi Shibata ${ }^{2}$, Fujiko Ando ${ }^{1,3}$ and Hiroshi Shimokata ${ }^{1,4}$ \\ ${ }^{1}$ Section of the NILS-LSA, National Center for Geriatrics and Gerontology, Aichi 474-8511, Japan \\ ${ }^{2}$ Institute for Health Care Science, Suntory Wellness Limited, Kyoto 619-0284, Japan \\ ${ }^{3}$ Faculty of Health and Medical Sciences, Aichi Shukutoku University, Aichi 480-1197, Japan \\ ${ }^{4}$ Graduate School of Nutritional Sciences, Nagoya University of Arts and Sciences, Aichi 470-0196, Japan \\ (Submitted 17 July 2015 - Final revision received 20 October 2015 - Accepted 2 November 2015 - First published online 22 December 2015)
}

\section{Abstract}

The effect of $n$-3 long-chain PUFA ( $n$-3 LCPUFA) on depression in healthy subjects is unclear, and most of the previous studies have focused on populations eating Western diets with lower fish intake. The present study investigated the association between blood levels of $n$ - 3 LCPUFA and depressive symptoms in Japanese community dwellers with higher $n-3$ LCPUFA blood levels. A cross-sectional study was conducted from 2006 to 2008, including 1050 men and 1073 women aged 40 years or older from the National Institute for Longevity Sciences the Longitudinal Study of Aging. The Center for Epidemiologic Studies Depression Scale (CES-D) was used to assess depressive symptoms. Multiple logistic regression analysis was performed to estimate the OR and $95 \%$ CI for a CES-D score $\geq 16$. Serum concentrations of $n$ - 3 PUFA, but not $n-6$ PUFA, were inversely associated with depressive symptoms. Compared with the lowest quintile, the adjusted OR for serum EPA at the fourth and fifth quintiles were 0.55 (95\% CI $0.35,0.85)$ and 0.64 (95\% CI $0.42,0.98)$, respectively, and at the fifth quintile for DHA it was 0.58 (95\% CI $0.37,0.92)$, for the presence of depressive symptoms ( $P_{\text {for trend }}=0.013$ and 0.011 , respectively). Serum levels of EPA and DHA were inversely associated with depressive symptoms in Japanese community dwellers with higher blood levels of $n$-3 LCPUFA, suggesting that n-3 LCPUFA intakes corresponding to higher levels in a Japanese population may have implications for a lower prevalence of depression.

Key words: $\boldsymbol{n}$-3 Long-chain PUFA: Depressive symptoms: Community-dwelling people: Japanese

Depression is a serious public health problem worldwide ${ }^{(1)}$. According to the seventeen nation World Mental Health Survey, approximately $5 \%$ of people report having experienced at least one episode of depression during their life ${ }^{(2)}$. By 2030, depression is predicted to become one of the top three causes of disability-adjusted life years lost ${ }^{(3)}$.

Management and prevention of depression are becoming increasingly important, especially for the middle-aged and the elderly. Depression in the middle-aged population results in economic losses and social burden; this social burden arises because the roles of middle-aged people in a community, including working and child rearing, keep growing. In addition, depressive symptoms are inter-related with multiple chronic conditions and age-related cognitive impairment ${ }^{(4)}$, as well as prodromal signs of cognitive decline in patients with early dementia $^{(5,6)}$. Thus, depression has attracted attention as a potential risk factor for dementia. Therefore, it should be recognised as a problem that needs to be addressed immediately in developed countries with ageing societies.
It is well known that diet and nutrition influence depression. EPA and DHA, the two major $n-3$ long-chain PUFA ( $n$-3 LCPUFA) derived from fish, are essential for the maintenance of cellular membrane functions via determination of membrane fluidity, and they affect neurotransmitter release and modify receptor binding ${ }^{(7)}$. Previous experimental studies have shown various mechanisms of these fatty acids, including anti-inflammatory $^{(8)}$, antioxidative ${ }^{(9)}$, neuroprotective ${ }^{(10)}$ and neurogenesis effects ${ }^{(11)}$. These factors may also be important in protecting against depression and its symptoms ${ }^{(12)}$. Thus, we hypothesised that $n-3$ LCPUFA may be able to influence the pathophysiology of depression.

According to a recent meta-analysis that took into account the heterogeneity among populations of randomised clinical trials, the use of $n$-3 LCPUFA was effective in patients with major depressive disorder (MDD) and in depressed patients without a diagnosis of $\mathrm{MDD}^{(13)}$. A meta-analysis of case-control studies, using blood levels as an indicator of $n-3$ LCPUFA levels, also reported significantly lower levels of EPA, DHA and total

Abbreviations: CES-D, Center for Epidemiologic Studies Depression Scale; $n$-3 LCPUFA, $n$-3 long-chain PUFA; NILS-LSA, National Institute for Longevity Sciences - the Longitudinal Study of Aging.

* Corresponding author: C. Horikawa, fax +81 77498 6262, email Chika_Noguchi@suntory.co.jp 
n-3 LCPUFA to be associated with depression ${ }^{(14)}$. These findings of associations in a general population are important in terms of preventing depression.

Previous ecological studies have shown that countries with high fish consumption, such as Japan, were associated with lower age-standardised disability-adjusted life-year rates for depressive disorders $^{(15)}$. However, studies on markers of dietary intake of $n-3$ LCPUFA in the same area/region provided contrasting results. To the best of our knowledge, only a few observational studies have examined the association between depression and n-3 LCPUFA, evaluated by measuring blood samples or intake estimation, in a general population with relatively high blood levels of $n-3$ LCPUFA $^{(16,17)}$. The lack of evidence regarding depressive symptoms in countries with high fish consumption may be explained in part by levels of $n$ - 3 LCPUFA being high enough to interact with the expression of depression.

The aim of our study was to investigate the association between blood levels of $n$ - 3 LCPUFA and depressive symptoms in a cross-sectional analysis of a community-dwelling population with higher $n$-3 LCPUFA blood levels. We specifically focused on middle-aged and elderly people, who have a higher risk for depression. We also examined other fatty acids comparatively, because it is necessary to ascertain whether findings regarding any association are specific to $n$-3 LCPUFA.

\section{Methods}

Data for the present study were obtained from the National Institute for Longevity Sciences - the Longitudinal Study of
Aging (NILS-LSA), a population-based survey of ageing in Japan. The participants in the NILS-LSA include about 2300 selected middle-aged and elderly subjects ${ }^{(18)}$. The normal ageing process has been researched over time using detailed questionnaires and medical check-ups, anthropometric measurements, physical fitness tests and nutritional assessments. Participants in the NILS-LSA included randomly selected age- and sex-stratified individuals from the pool of non-institutionalised residents of the National Center for Geriatrics and Gerontology's neighbourhood areas of Obu City and Higashiura Town in Aichi Prefecture. Details of the NILS-LSA study have been reported elsewhere ${ }^{(18)}$.

\section{Subjects}

Subjects in this cross-sectional analysis were derived from the fifthwave examination of the NILS-LSA. The fifth-wave examination, which comprehensively measured various fatty acids, comprised 2419 participants aged 40 years and older, and was started in July 2006 and completed in July 2008. After the exclusion of 296 subjects because of fasting for $<12 \mathrm{~h}$ ( $n 53$ ), history of and current dementia ( $n$ 6) or lack of available data for analysis ( $n$ 237), a total of 2123 subjects (1050 men, 1073 women) were included in the analysis (Fig. 1). Self-reported history of previously diagnosed dementia was collected at the fifth-wave interview.

The study was approved by the Committee for Ethics of Human Research of the National Center for Geriatrics and Gerontology (no. 249). Written informed consent was obtained from all the subjects.

$$
\begin{aligned}
& \text { Community-living population aged } 40 \text { years and older in the } \\
& \text { neighbourhood area of the National Institute for Longevity Sciences } \\
& \text { Obu City (population approximately } 79000 \text { ) } \\
& \text { Higashiura Town (population approximately } 48000 \text { ) }
\end{aligned}
$$

Stratified by both age and sex and randomly selected from resident registrations

Participants (provided informed consent) ( $n$ 2419)

in the fifth-wave examination from July 2006 to July 2008

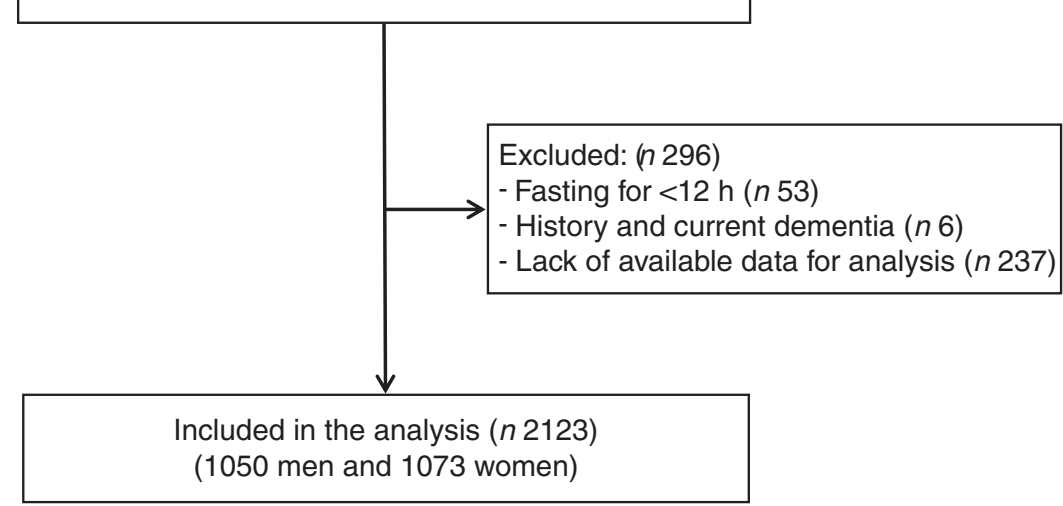

Fig. 1. Flow chart for the individuals included in the study. 


\section{Serum fatty acid levels}

Venous blood was collected early in the morning after at least $12 \mathrm{~h}$ of fasting. Blood samples were centrifuged at $3500 \mathbf{g}$ for $15 \mathrm{~min}$. Serum was separated and immediately frozen at $-80^{\circ} \mathrm{C}$. Total lipids in serum were extracted and purified by the Folch procedure. Serum fatty acid concentration was measured using a GC at a clinical laboratory (SRL). In brief, fatty acid residues in the lipids were transmethylated and analysed using a GC (GC-17A; Shimadzu) with a capillary column Omegawax 250 (Supelco). Absolute amounts of each fatty acid were quantified by standards as weight concentrations $(\mu \mathrm{g} / \mathrm{ml})$.

\section{Depressive symptoms}

Depressive symptoms were assessed using the Japanese version $^{(19)}$ questionnaire of the Center for Epidemiologic Studies Depression Scale (CES-D) ${ }^{(20)}$. Participants completed a CES-D questionnaire, which assessed the depressed state during a previous week, approximately 2 weeks before each exploratory visit for the fifth-wave examination. The CES-D questionnaire comprises twenty questions in four subscales: somatic and retarded activity, depressed affect, positive affect and interpersonal relations. Scores range from 0 to 60 , with lower scores indicating fewer depressive symptoms. This scale has been reported to be a valid and reliable measure of depressive symptoms in the elderly ${ }^{(21)}$. A cut-off score of $\geq 16$ is used to identify subjects with relevant depressive symptoms ${ }^{(20)}$. We defined a CES-D score $\geq 16$ as representative of depressive symptoms.

\section{Other measurements}

Data regarding medical history of hypertension, hyperlipidaemia, ischaemic heart disease, stroke, diabetes and dementia (yes/no), education ( $\leq 9,10-12$, or $\geq 13$ years of school), employment status (no occupation or household labour/ non-regular employment/regular employment), marital status (yes/no), sex, age and smoking status (yes/no) were asked in the survey questionnaires. This information was obtained via a self-completed questionnaire provided approximately 2 weeks before the exploratory visit; questionnaires were collected on the same day as blood samples. BMI was calculated as weight in kilograms divided by the square of height in metres $\left(\mathrm{kg} / \mathrm{m}^{2}\right)$. Both weight and height were actual measured values obtained on the same day that blood samples were collected. Alcohol intake $(\mathrm{ml} / \mathrm{d})$ during the previous year was assessed using a FFQ through interviews with well-trained dietitians. The number of steps walked per day was used to assess physical activity. Participants wore a uniaxial accelerometry sensor (Lifecorder; Suzuken) for a 7-d period (except while sleeping or bathing) for 2 weeks after the exploratory visit. We calculated the mean number of steps from 5 -d records (the maximum and minimum values were excluded).

\section{Statistical analysis}

All the statistical analyses were conducted using Statistical Analysis System version 9.3 (SAS Institute). The confounding variables were sex, age, BMI, education level, marital status, smoking status, alcohol consumption, physical activity, employment status and history of hypertension, hyperlipidaemia, ischaemic heart disease, stroke and diabetes. Differences in characteristics and serum fatty acid concentrations between subjects with and without depressive symptoms were assessed using the $\chi^{2}$ test (categorical variables) and Student's $t$ test (continuous variables). For analysis of the association between serum fatty acid concentrations and depressive symptoms, we carried out multiple logistic regression analysis and estimated the OR and $95 \%$ CI of depressive symptoms for quintiles of serum fatty acid concentrations, using the lowest quintile category as the reference. Trend associations were assessed by entering dummy variables assigned to the quintile of serum fatty acid concentrations. In model I, we adjusted for age and sex. Model II was further adjusted for the above-described confounding variables. A sub-analysis by sex also was performed for EPA and DHA. Two-sided $P$ values $<0.05$ were regarded as statistically significant.

\section{Results}

The study subjects included 1050 men (49.5\%) and 1073 women $(50 \cdot 5 \%)$. In total, the mean age was $60 \cdot 3$ (SD $12 \cdot 3$ ) years, and 266 subjects $(12.5 \%)$ were identified as having depressive symptoms (CES-D scores $\geq 16$ ). Characteristics according to subjects with and without depressive symptoms are shown in Table 1. Subjects with depressive symptoms were significantly more likely to be unmarried, have fewer years of education and have a higher rate of past stroke compared with subjects without depressive symptoms.

Serum concentrations of principal fatty acids based on the presence or absence of depressive symptoms are shown in Table 2. There were no significant differences in any serum fatty acid concentrations between groups. However, several fatty acids showed marginally significant differences between subjects with and without depressive symptoms, including EPA $(P=0 \cdot 068), n$-3 PUFA $(P=0 \cdot 072)$ and $n$-3 LCPUFA $(P=0 \cdot 078)$. The mean $n-3$ LCPUFA concentrations of subjects with and without depressive symptoms were $264 \cdot 1$ (sD 101.2) $\mu \mathrm{g} / \mathrm{ml}$ and $276 \cdot 0$ (sD 103.4) $\mu \mathrm{g} / \mathrm{ml}$, respectively.

Tables 3 and 4 present the OR and 95\% CI of depressive symptoms for quintiles of serum fatty acid concentrations, using the lowest quintile category as the reference. In Table 3, SFA, MUFA, PUFA and $n-6$ PUFA were not significant, but the $n-3$ : $n$ - 6 ratio partially and $n$ - 3 PUFA showed an inverse association in all models. Individual fatty acids of the PUFA series are shown in Table 4. In the crude model, EPA and DHA showed an inverse association with depressive symptoms $\left(P_{\text {for trend }}=\right.$ 0.021 and 0.039 , respectively). The crude OR for EPA at the fourth quintile was lower than that for the lowest quintile (OR 0.58 ; $95 \%$ CI $0.38,0.88$ ). In Model I, adjusting for sex and age, the OR for EPA at the fourth and fifth quintiles and for DHA at the fifth quintile were significantly lower compared with reference values. Furthermore, these significant associations were maintained, even after adjusting for more covariates including age, sex, BMI, education level, marital status, smoking status, alcohol consumption, physical activity, employment status and history of hypertension, hyperlipidaemia, ischaemic heart 
Table 1. Characteristics of subjects with and without depressive symptoms (Numbers and percentages; mean values and standard deviations)

\begin{tabular}{|c|c|c|c|c|c|}
\hline \multirow[b]{2}{*}{ Variables } & \multicolumn{2}{|c|}{$\begin{array}{l}\text { Subjects without depressive symptoms } \\
\qquad(\text { CES-D <16) }\end{array}$} & \multicolumn{2}{|c|}{$\begin{array}{l}\text { Subjects without depressive symptoms } \\
\qquad(\text { CES-D } \geq 16)\end{array}$} & \multirow[b]{2}{*}{$P^{*}$} \\
\hline & $n$ & $\%$ & $n$ & $\%$ & \\
\hline Number of subjects & 1857 & & 266 & & \\
\hline Sex (male) & 933 & $50 \cdot 2$ & 117 & $44 \cdot 0$ & 0.056 \\
\hline Age (years) & & & & & 0.122 \\
\hline Mean & \multicolumn{2}{|c|}{$60 \cdot 1$} & \multicolumn{2}{|c|}{61.4} & \\
\hline $\mathrm{SD}$ & \multicolumn{2}{|c|}{$12 \cdot 3$} & \multicolumn{2}{|c|}{$12 \cdot 8$} & \\
\hline $\mathrm{BMI}\left(\mathrm{kg} / \mathrm{m}^{2}\right)$ & & & & & 0.071 \\
\hline Mean & \multicolumn{2}{|c|}{22.9} & \multicolumn{2}{|c|}{$22 \cdot 5$} & \\
\hline SD & \multicolumn{2}{|c|}{3.0} & \multicolumn{2}{|c|}{$3 \cdot 2$} & \\
\hline CES-D & & & & & $<0.001$ \\
\hline Mean & \multicolumn{2}{|c|}{$5 \cdot 3$} & \multicolumn{2}{|c|}{21.9} & \\
\hline SD & \multicolumn{2}{|c|}{4.4} & \multicolumn{2}{|c|}{$6 \cdot 2$} & \\
\hline \multicolumn{6}{|l|}{ Education level } \\
\hline$\leq 9$ years & 329 & $17 \cdot 7$ & 58 & $21 \cdot 8$ & 0.038 \\
\hline $10-12$ years & 738 & $39 \cdot 7$ & 116 & $43 \cdot 6$ & \\
\hline$\geq 13$ years & 790 & $42 \cdot 5$ & 92 & $34 \cdot 6$ & \\
\hline Unmarried & 254 & $13 \cdot 7$ & 72 & $27 \cdot 1$ & $<0.001$ \\
\hline Current smoker & 262 & $14 \cdot 1$ & 45 & $16 \cdot 9$ & 0.223 \\
\hline Alcohol consumption† (ml/d) & & & & & 0.842 \\
\hline Mean & \multicolumn{2}{|c|}{0.37} & \multicolumn{2}{|c|}{0.35} & \\
\hline SD & \multicolumn{2}{|c|}{$2 \cdot 61$} & \multicolumn{2}{|c|}{1.96} & \\
\hline Physical activity (steps/d) & & & & & $0 \cdot 274$ \\
\hline Mean & \multicolumn{2}{|c|}{8746} & \multicolumn{2}{|c|}{8500} & \\
\hline $\mathrm{SD}$ & \multicolumn{2}{|c|}{3417} & \multicolumn{2}{|c|}{3439} & \\
\hline \multicolumn{6}{|l|}{ Employment } \\
\hline Unemployed or household labour & 766 & $41 \cdot 3$ & 123 & $46 \cdot 2$ & 0.207 \\
\hline Non-regular employment & 385 & $20 \cdot 7$ & 56 & $21 \cdot 1$ & \\
\hline Regular employment & 706 & $38 \cdot 0$ & 87 & $32 \cdot 7$ & \\
\hline \multicolumn{6}{|l|}{ Medical history $\ddagger$} \\
\hline Stroke & 53 & $2 \cdot 9$ & 16 & $6 \cdot 0$ & 0.007 \\
\hline Hypertension & 513 & $27 \cdot 6$ & 82 & $30 \cdot 8$ & 0.277 \\
\hline Ischaemic heart disease & 74 & $4 \cdot 0$ & 13 & 4.9 & 0.488 \\
\hline Hyperlipidaemia & 349 & $18 \cdot 8$ & 59 & $22 \cdot 2$ & 0.190 \\
\hline Diabetes mellitus & 134 & $7 \cdot 2$ & 21 & $7 \cdot 9$ & 0.691 \\
\hline
\end{tabular}

CES-D, Center for Epidemiologic Studies Depression Scale.

* Continuous variables: Student's $t$ test, Categorical variables: $x^{2}$ test.

$\dagger$ Converting alcohol consumption into ethanol content.

‡ Past and present illness.

Table 2. Serum fatty acid concentration $(\mu \mathrm{g} / \mathrm{ml})$ and ratio of study subjects (Mean values and standard deviations)

\begin{tabular}{|c|c|c|c|c|c|}
\hline & \multicolumn{2}{|c|}{ Subjects without depressive symptoms (CES-D $<16)$} & \multicolumn{2}{|c|}{ Subjects with depressive symptoms (CES-D $\geq 16$ ) } & \multirow[b]{2}{*}{$P^{*}$} \\
\hline & Mean & SD & Mean & SD & \\
\hline SFA & $1001 \cdot 8$ & $270 \cdot 6$ & 991.9 & 254.9 & 0.571 \\
\hline MUFA & $707 \cdot 3$ & $260 \cdot 9$ & $710 \cdot 9$ & $222 \cdot 1$ & 0.807 \\
\hline PUFA & $1454 \cdot 2$ & 274.9 & $1429 \cdot 7$ & $250 \cdot 7$ & 0.170 \\
\hline$n-3$ PUFA & 303.9 & $110 \cdot 6$ & $290 \cdot 9$ & $105 \cdot 9$ & 0.072 \\
\hline DHA & 174.0 & 58.4 & $168 \cdot 2$ & $57 \cdot 3$ & 0.125 \\
\hline EPA & 78.8 & $44 \cdot 1$ & 73.5 & $43 \cdot 4$ & 0.068 \\
\hline ALA & 27.9 & 19.5 & $26 \cdot 8$ & $12 \cdot 2$ & 0.218 \\
\hline$n-6$ PUFA & $1148 \cdot 7$ & $222 \cdot 5$ & $1137 \cdot 1$ & $199 \cdot 6$ & 0.385 \\
\hline ARA & $174 \cdot 7$ & $40 \cdot 1$ & $174 \cdot 0$ & $39 \cdot 5$ & 0.786 \\
\hline LA & $915 \cdot 8$ & $192 \cdot 0$ & $905 \cdot 2$ & $174 \cdot 1$ & 0.359 \\
\hline$n-3: n-6$ & 0.270 & 0.101 & 0.260 & 0.095 & 0.143 \\
\hline
\end{tabular}

CES-D, Center for Epidemiologic Studies Depression Scale; $n$-3 PUFA, sum of ALA, EPA, DPA and DHA; ALA, $a$-linolenic acid; $n$-6 PUFA, sum of LA $\gamma$-linolenic acid, eicosadienoic acid, dihomo- $\gamma$-linolenic acid, ARA and docosatetraenoic acid; ARA, arachidonic acid; LA, linoleic acid.

* Student's $t$ test.

disease, stroke and diabetes. In Model II, after adjusting for all covariates, EPA was associated with a lower OR of depressive symptoms (fourth quartile $v$. lowest: OR 0.55; $95 \%$ CI 0.35, 0.85; fifth quartile $v$. lowest: OR 0.64; $95 \%$ CI $0.42,0.98 ; P_{\text {for trend }}=$ 0.013). Similarly, the quintile of DHA was associated with a lower OR of depressive symptoms (fifth quartile $v$. lowest: 


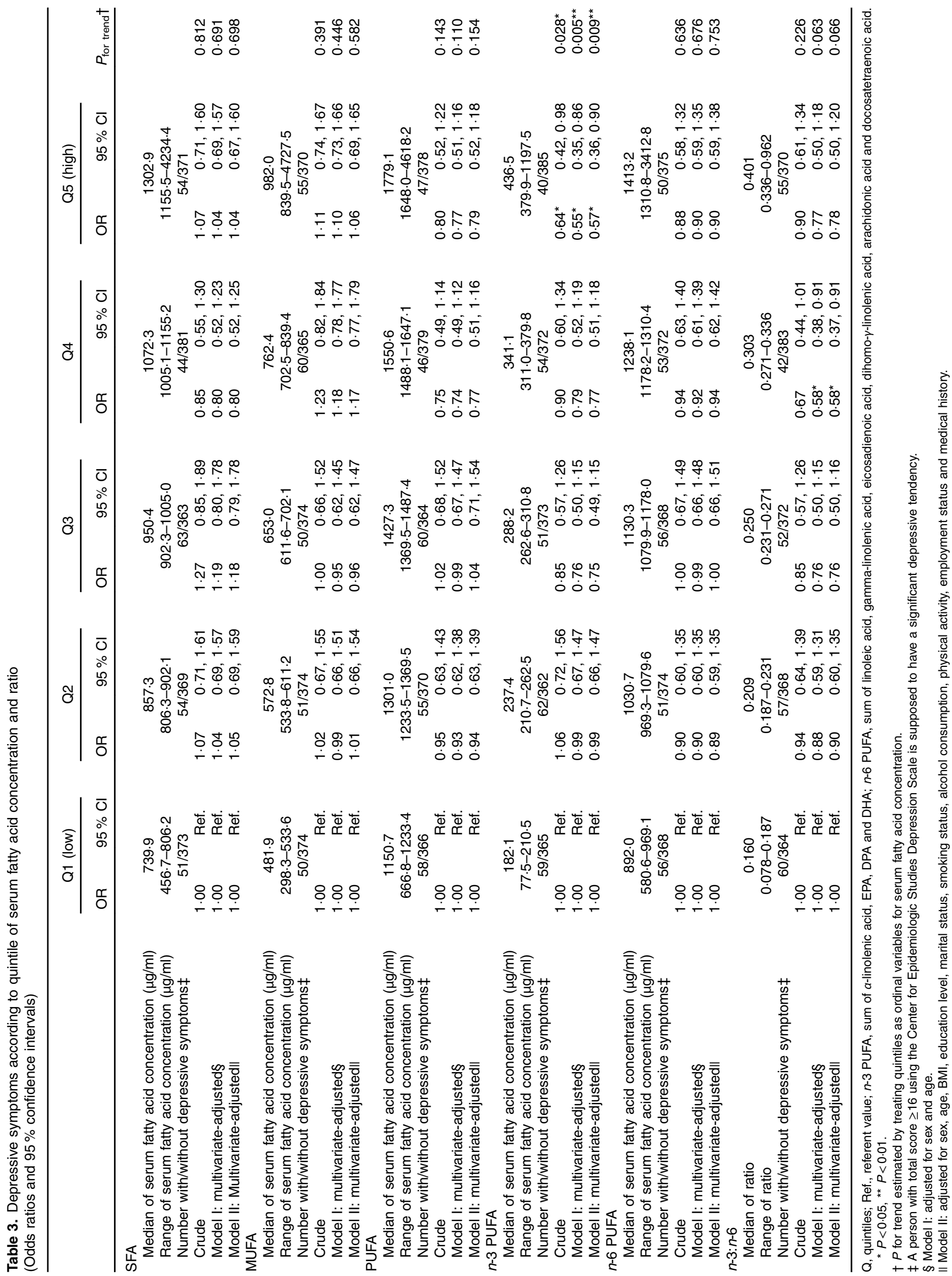


*ั*

ำ 눙ํำ

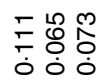

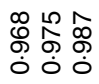

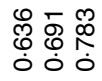

잉요

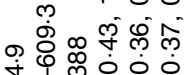

委京誉

@令

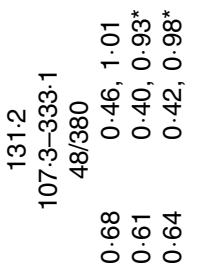

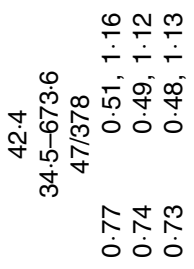

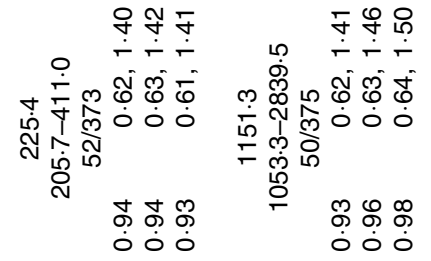

ब्रूल

$\infty \infty \infty$

으 웅

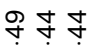

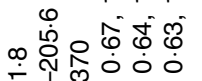

ब은

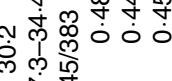

o

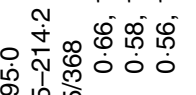

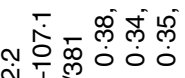

ช่ $\frac{1}{\circ}$

$\begin{array}{lll}\infty & & \\ 0 & 0 & 0 \\ 0 & 0 & 0 \\ 0 & 0 & 0\end{array}$

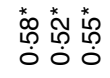

ํํㅇํㅇ옹

요용

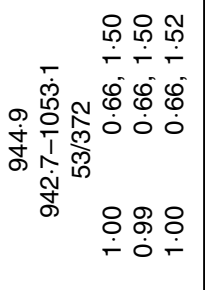

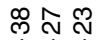

ำ 뉴 는

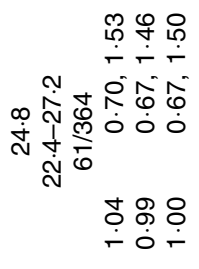

กิ้

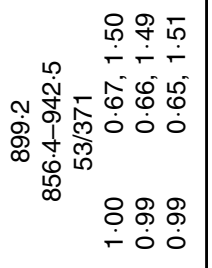

ำ

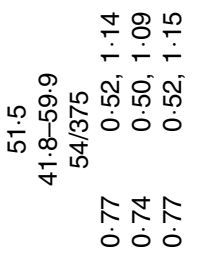

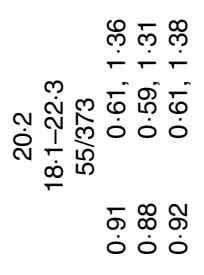

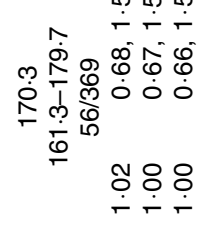

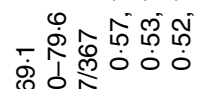

远

$\begin{array}{lll}\text { m} & \infty \\ 0 & \stackrel{1}{0} \\ 0 & 0 & 0\end{array}$

$\dot{+} \dot{0} \dot{+}$

จิ ลิ

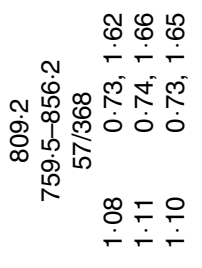

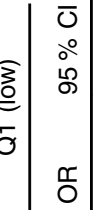

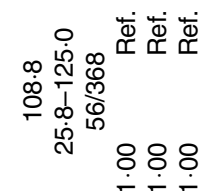

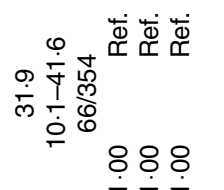

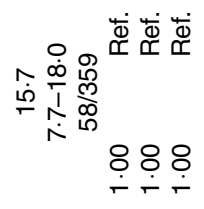

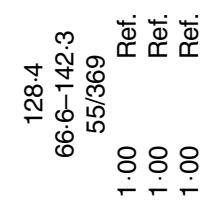

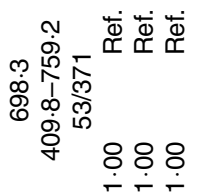

๑ั

范

$\begin{array}{lll}0 & 1 \\ 0 & 0 \\ 0 & 0 & 0 \\ 0 & 0 & 0\end{array}$

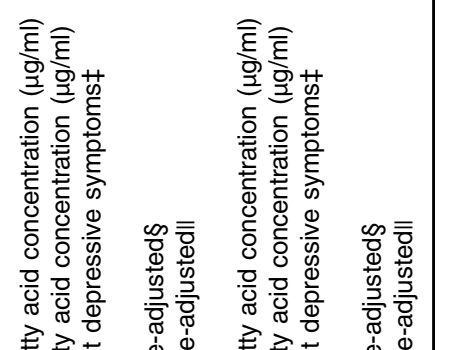

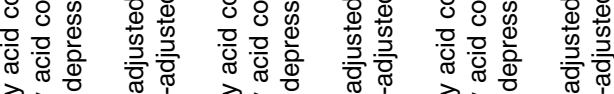

हิ
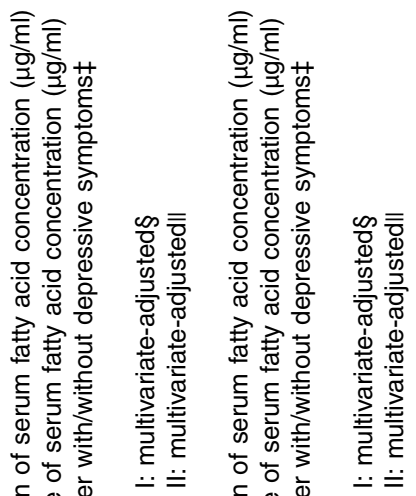

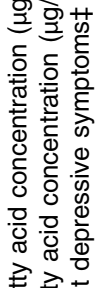

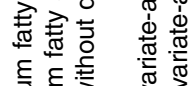

害言吉

空焉

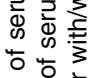

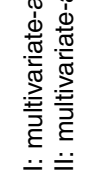


OR 0.58; $95 \%$ CI 0.37, 0.92; $P_{\text {for trend }}=0.011$ ). There were no significant associations for other fatty acids. In addition, a sub-analysis by sex was performed for EPA and DHA. In women only, EPA showed a significant association with a lower OR of depressive symptoms in model II (fourth quartile $v$. lowest: OR 0.47; $95 \%$ CI 0.26, 0.86; fifth quartile $v$. lowest: OR $0.51 ; 95 \%$ CI $\left.0.28,0.93 ; P_{\text {for trend }}=0.013\right)$. There were no significant associations for DHA in both sexes.

\section{Discussion}

In the present study, inverse associations between serum EPA and DHA concentrations and depressive symptoms were observed in Japanese middle-aged and elderly communitydwelling subjects. This is the first study demonstrating the inverse association in community-dwelling Japanese people with higher blood levels of $n$-3 LCPUFA. No positive association for other fatty acids indicates that this association is specific to $n$-3 LCPUFA

The prevalence of depressive symptoms among subjects was $12.5 \%$ in this study. In another survey of nearly 5000 Japanese elderly aged 65 years and older, Yokoyama et al. ${ }^{(22)}$ showed that the prevalence of depression was $13.8 \%$. The incidence rate is approximately the same as the present study, although depression in their study was evaluated according to the eleven-item short form of the CES-D.

Subjects in the present study were Japanese and had higher serum levels of $n$ - 3 LCPUFA compared with populations in Western countries ${ }^{(23-25)}$. Median values for serum EPA, DHA and $n$-3 LCPUFA were $69 \cdot 1,165 \cdot 6$ and $261 \cdot 3 \mu \mathrm{g} / \mathrm{ml}$, respectively. Japanese consume a large amount of fish and seafood ${ }^{(26)}$, the main sources of $n$-3 LCPUFA $^{(24)}$, and $n$-3 LCPUFA intake correlates well with blood levels ${ }^{(27)}$. In a previous study, Japanese middle-aged and elderly subjects also showed similar levels of serum $n-3$ LCPUFA $^{(28)}$. In American middle-aged and elderly subjects with hypercholesterolaemia ( $n$ 105), the median values for serum EPA and DHA were 18 and $46 \mu \mathrm{g} / \mathrm{ml}$, respectively $^{(29)}$. In Brazilian middle-aged subjects ( $<50$ years), the median value for serum $n-3$ LCPUFA was $150 \mu \mathrm{g} / \mathrm{ml}^{(30)}$. Although not a stringent comparison because we determined non-fractionated and serum concentrations in our study, the mean compositions for erythrocyte phospholipids of EPA and DHA in another Japanese elderly population were 2.3 and $6.7 \%$, respectively ${ }^{(31)}$, and 0.3 and $3.7 \%$ in elderly Italian females ${ }^{(32)}$. The $n$-3 LCPUFA blood level in Japanese subjects is more than two times higher compared with that of subjects from Western countries.

A recent meta-analysis was conducted in countries with populations with lower blood levels of $n-3$ LCPUFA, and the results showed lower blood levels of $n$-3 LCPUFA in patients with depression ${ }^{(14)}$. An inverse association between serum n-3 LCPUFA levels and depressive symptoms in communitydwelling people with higher blood levels of $n$-3 LCPUFA has not been observed so far. We considered that the lack of evidence regarding depressive symptoms in populations with higher blood levels of $n$-3 LCPUFA may depend on levels of $n$-3 LCPUFA being high enough to interact with the expression of depression. However, we found significant trends for these inverse associations between serum $n$ - 3 LCPUFA and depressive symptoms in the present study.

To the best of our knowledge, only two studies investigated the association between serum levels of $n-3$ LCPUFA and depressive symptoms in Korean or Japanese subjects with high blood levels of $n$-3 LCPUFA $^{(16,17)}$. In the Japanese study, no association was observed between blood $n$-3 LCPUFA levels and depressive symptoms, whereas a significant association was noted in Korean subjects. The Korean study used a case-control design with eighty depressive patients and eighty controls, including a higher percentage of women. On the other hand, the Japanese study included 113 healthy men. The composition ratio of sex may partially influence results. According to three previous studies of dietary intervention in Western countries, the association appears to be observed more in women than in men ${ }^{(33-35)}$. The NILS-LSA is a cohort study that has approximately 280 subjects in each group classified by age decade and sex, and thus roughly half the subjects are women. We showed a significant association for EPA in women through a sub-analysis stratified by sex, and assumed higher endogenous $n$ - 3 LCPUFA in women because of oestrogenic effects as a reasonable possibility for this sex difference ${ }^{(36)}$. However, this finding should be interpreted with caution in light of evidence from a previous report implicating the stronger association in men than in women between $n$-3 LCPUFA intakes and the occurrence of depressive episodes ${ }^{(37)}$

The Korean study was conducted in patients with depression $^{(17)}$, but subjects in the Japanese study were healthy ${ }^{(16)}$. Most previous studies showing a significant association between $n-3$ LCPUFA and depressive symptoms were conducted in depressed patients ${ }^{(13,14)}$. However, subjects who participated in the NILSLSA were non-hospitalised, community-dwelling people, and we did not have a diagnosis of clinical depression. There might be other reasons for our findings.

Our findings may be due to the characteristics and the design of the NILS-LSA. Participants in the NILS-LSA included randomly selected age- and sex-stratified individuals; therefore, they were equally distributed to each group by generation and sex. In contrast, most studies investigated more limited samples. The 113 healthy men in the Japanese study represented a limited sample from the same workplace and a similar younger generation. It might be that the equal proportion size of each age- and sex-stratified group in the present study led to the significant results. There is indeed a $75 \%$ power to detect the significance for serum EPA at the highest quintile, but the DHA power is only $48 \%$ (POWER procedure, SAS version 9.3). Most fatty acids also show low power. For fatty acids other than EPA and DHA, we consider that a failure to detect an effect was present.

The most relevant reason for the new findings in our study is that the NILS-LSA has the ability to adjust for confounding factors. Medical history including stroke and diabetes is recognised as a critical complication of depression ${ }^{(38-40)}$, although medical history was not adjusted for in the Japanese study mentioned above ${ }^{(16)}$. As many individual characteristics may be involved in the development of depressive symptoms and ageing, study results should be interpreted after adjusting for confounding factors. 
There were a few differences between EPA and DHA in the present study, as the quintiles of both fatty acid concentrations showed an association with a lower OR for depressive symptoms, with significant trends. Other reports have indicated the possibility of a better effect of EPA than of DHA on depression $^{(41,42)}$. A recent meta-analysis provided evidence that EPA may be more efficacious than DHA in treating depression ${ }^{(43)}$. Another meta-analysis also suggested that only regimens containing over $60 \%$ EPA showed a highly significant effect in the treatment of depression ${ }^{(42)}$. DHA is also a main component of brain neurons ${ }^{(44)}$ and plays an important role in maintaining regular brain function ${ }^{(45)}$. An intervention study and a metaanalytic review indicated the possibility of a better effect of DHA than EPA on depression ${ }^{(14,46)}$

This study has several limitations. First, it is not possible to prove a causal relationship between serum fatty acid concentrations and depressive symptoms because this study is a cross-sectional analysis. The association can be potentially explained by the possibility that depressive symptoms may lead to a lower intake of $n$-3 LCPUFA through decreased appetite and food consumption and a reduction in activity. Further longitudinal studies are needed to assess this relationship.

Second, we did not make a diagnosis of clinical depression in this study. Although the CES-D is a valuable assessment scale to study the relationship between depressive symptoms and several variables, it is not a clinical diagnostic tool ${ }^{(20)}$.

In conclusion, the present study suggests that the serum levels of EPA and DHA may be associated with depressive symptoms in Japanese with higher blood levels of $n-3$ LCPUFA. Therefore, even in countries with a higher fish intake, $n-3$ LCPUFA intake in the usual diet may lower depressive symptoms. Furthermore, the efficacy of $n-3$ LCPUFA against depressive symptoms may be present in Japanese community dwellers. Longitudinal and intervention studies are needed to elucidate any protective effects of $n-3$ LCPUFA against depression.

\section{Acknowledgements}

The authors express their sincere appreciation to the study participants and their colleagues in the NILS-LSA for completing the survey for this study.

This study did not receive any financial funding.

Conceived the idea and designed the study: C. H., R. O., F. A. and H. Shimokata. Data collection: H. Shimokata, F. A., R. O., Y. K., Y. N. and C. T. Performed the analysis: C. H. Interpreted the data: C. H., H. Shimokata, F. A., R. O., T. R. and S. K. Drafted the manuscript: C. H., R. O., S. K. and T. R. Critical revision of manuscript for important intellectual content: H. Shimokata, F. A., R. O., H. Shibata and H. K.

Five of the authors (C. H., S. K., T. R., H. K. and H. Shibata) are employees of Suntory Wellness Limited. This does not alter the authors' adherence to all the journal policies.

\section{References}

1. Doris A, Ebmeier K \& Shajahan P (1999) Depressive illness. Lancet 354, 1369-1375.
2. World Health Organization (2012) World mental health day 2012 - depression: a global crisis. http://www.who.int/mental_ health/management/depression/en/ (accessed February 2014).

3. World Health Organization (2014) Health topics/depression. http://www.emro.who.int/health-topics/depression/index.html (accessed Feburuary 2014).

4. Kesse-Guyot E, Touvier M, Andreeva VA, et al. (2012) Crosssectional but not longitudinal association between $n-3$ fatty acid intake and depressive symptoms: results from the SU.VI. MAX 2 study. Am J Epidemiol 175, 979-987.

5. Green RC, Cupples LA, Kurz A, et al. (2003) Depression as a risk factor for Alzheimer disease: the MIRAGE Study. Arch Neurol 60, 753-759.

6. Ownby RL, Crocco E, Acevedo A, et al. (2006) Depression and risk for Alzheimer disease: systematic review, meta-analysis, and metaregression analysis. Arch Gen Psychiatry 63, 530-538.

7. Hulbert AJ, Turner N, Storlien LH, et al. (2005) Dietary fats and membrane function: implications for metabolism and disease. Biol Rev Camb Philos Soc 80, 155-169.

8. Simopoulos AP (2002) Omega-3 fatty acids in inflammation and autoimmune diseases. J Am Coll Nutr 21, 495-505.

9. Liu Q, Wu D, Ni N, et al. (2014) Omega-3 polyunsaturated fatty acids protect neural progenitor cells against oxidative injury. Mar Drugs 12, 2341-2356.

10. Hong SH, Belayev L, Khoutorova L, et al. (2014) Docosahexaenoic acid confers enduring neuroprotection in experimental stroke. J Neurol Sci 338, 135-141.

11. Kang JX \& Gleason ED (2013) Omega-3 fatty acids and hippocampal neurogenesis in depression. CNS Neurol Disord Drug Targets 12, 460-465.

12. Stewart JC, Rand KL, Muldoon MF, et al. (2009) A prospective evaluation of the directionality of the depression-inflammation relationship. Brain Behav Immun 23, 936-944.

13. Grosso G, Pajak A, Marventano S, et al. (2014) Role of omega-3 fatty acids in the treatment of depressive disorders: a comprehensive meta-analysis of randomized clinical trials. PLOS ONE 9, e96905.

14. Lin PY, Huang SY \& Su KP (2010) A meta-analytic review of polyunsaturated fatty acid compositions in patients with depression. Biol Psychiatry 68, 140-147.

15. Grosso G, Galvano F \& Marventano S (2014) Omega-3 fatty acids and depression: scientific evidence and biological mechanisms. Oxid Med Cell Longev 2014, 313570.

16. Tsuboi H, Watanabe M, Kobayashi F, et al. (2013) Associations of depressive symptoms with serum proportions of palmitic and arachidonic acids, and $\alpha$-tocopherol effects among male population - a preliminary study. Clin Nutr 32, 289-293.

17. Baek D \& Park Y (2013) Association between erythrocyte $n-3$ polyunsaturated fatty acids and biomarkers of inflammation and oxidative stress in patients with and without depression. Prostaglandins Leukot Essent Fatty Acids 89, 291-296.

18. Shimokata H, Ando F \& Niino N (2000) A new comprehensive study on aging - the National Institute for Longevity Sciences, Longitudinal Study of Aging (NILS-LSA). J Epidemiol 10, S1-S9.

19. Shima S, Shikano T, Kitamura T, et al. (1985) Atarashii yokuutsusei jiko hyouka syakudo ni tuite [New self-rating scale for depression]. Clin Psychiatry 27, 717-723.

20. Radloff LS (1977) The CES-D scale: a self-report depression scale for research in the general population. Appl Psychol Meas 1, 385-401.

21. Berkman LF, Berkman CS, Kasl S, et al. (1986) Depressive symptoms in relation to physical health and functioning in the elderly. Am J Epidemiol 124, 372-388. 
22. Yokoyama E, Kaneita Y, Saito Y, et al. (2010) Association between depression and insomnia subtypes: a longitudinal study on the elderly in Japan. Sleep 33, 1693-1702.

23. Kobayashi M, Sasaki S, Kawabata T, et al. (2001) Single measurement of serum phospholipid fatty acid as a biomarker of specific fatty acid intake in middle-aged Japanese men. Eur J Clin Nutr 55, 643-650.

24. Elmadfa I \& Kornsteiner M (2009) Fats and fatty acid requirements for adults. Ann Nutr Metab 55, 56-75.

25. Otsuka R, Kato Y, Imai T, et al. (2013) Higher serum EPA or DHA, and lower ARA compositions with age independent fatty acid intake in Japanese aged 40 to 79 . Lipids $\mathbf{4 8}, 719-727$.

26. Miyagawa N, Miura K, Okuda N, et al. (2014) Long-chain n-3 polyunsaturated fatty acids intake and cardiovascular disease mortality risk in Japanese: a 24-year follow-up of NIPPON DATA80. Atherosclerosis 232, 384-389.

27. Nakamura T, Azuma A, Kuribayashi T, et al. (2003) Serum fatty acid levels, dietary style and coronary heart disease in three neighbouring areas in Japan: the Kumihama study. $\mathrm{BrJ}$ Nutr 89, 267-272.

28. Conklin SM, Harris JI, Manuck SB, et al. (2007) Serum omega-3 fatty acids are associated with variation in mood, personality and behavior in hypercholesterolemic community volunteers. Psychiatry Res 152, 1-10.

29. Fekete K, Marosvölgyi T, Jakobik V, et al. (2009) Methods of assessment of $n-3$ long-chain polyunsaturated fatty acid status in humans: a systematic review. Am J Clin Nutr 89, 2070S-2084S

30. Kobayashi M, Sasaki S, Hamada GS, et al. (1999) Serum n-3 fatty acids, fish consumption and cancer mortality in six Japanese populations in Japan and Brazil. Jpn J Cancer Res 90, 914-921.

31. Kawabata T, Hirota S, Hirayama T, et al. (2011) Age-related changes of dietary intake and blood eicosapentaenoic acid, docosahexaenoic acid, and arachidonic acid levels in Japanese men and women. Prostaglandins Leukot Essent Fatty Acids 84, 131-137.

32. Rondanelli M, Giacosa A, Opizzi A, et al. (2011) Long chain omega 3 polyunsaturated fatty acids supplementation in the treatment of elderly depression: effects on depressive symptoms, on phospholipids fatty acids profile and on healthrelated quality of life. J Nutr Health Aging. 15, 37-44.

33. Lespérance F, Frasure-Smith N, St-André E, et al. (2011) The efficacy of omega-3 supplementation for major depression: a randomized controlled trial. J Clin Psychiatry $\mathbf{7 2}$, 1054-1062.

34. Colangelo LA, He K, Whooley MA, et al. (2009) Higher dietary intake of long-chain omega-3 polyunsaturated fatty acids is inversely associated with depressive symptoms in women. Nutrition 25, 1011-1019.

35. Sanchez-Villegas A, Henríquez P, Figueiras A, et al. (2007) Long chain omega- 3 fatty acids intake, fish consumption and mental disorders in the SUN cohort study. Eur J Nutr $\mathbf{4 6}$, 337-346.

36. Giltay EJ, Gooren LJ, Toorians AW, et al. (2004) Docosahexaenoic acid concentrations are higher in women than in men because of estrogenic effects. Am J Clin Nutr. 80, $1167-1174$

37. Astorg P, Couthouis A, Bertrais S, et al. (2008) Association of fish and long-chain $n-3$ polyunsaturated fatty acid intakes with the occurrence of depressive episodes in middle-aged French men and women. Prostaglandins Leukot Essent Fatty Acids 78, 171-182

38. Lindén T, Blomstrand C \& Skoog I. (2007) Depressive disorders after 20 months in elderly stroke patients: a casecontrol study. Stroke 38, 1860-1863.

39. de Jonge P, Roy JF, Saz P, et al. (2006) Prevalent and incident depression in community-dwelling elderly persons with diabetes mellitus: results from the ZARADEMP project. Diabetologia 49, 2627-2633.

40. Maraldi C, Volpato S, Penninx BW, et al. (2007) Diabetes mellitus, glycemic control, and incident depressive symptoms among 70- to 79-year-old persons: the health, aging, and body composition study. Arch Intern Med 167, 1137-1144.

41. Bloch MH \& Hannestad J (2012) Omega-3 fatty acids for the treatment of depression: systematic review and meta-analysis. Mol Psychiatry 17, 1272-1282.

42. Martins JG, Bentsen H \& Puri BK (2012) Eicosapentaenoic acid appears to be the key omega-3 fatty acid component associated with efficacy in major depressive disorder: a critique of Bloch and Hannestad and updated meta-analysis. Mol Psychiatry 17, 1144-1149.

43. Martins JG (2009) EPA but not DHA appears to be responsible for the efficacy of omega-3 long chain polyunsaturated fatty acid supplementation in depression: evidence from a metaanalysis of randomized controlled trials. J Am Coll Nutr 28, 525-542.

44. Wainwright PE (2002) Dietary essential fatty acids and brain function: a developmental perspective on mechanisms. Proc Nutr SOC 61, 61-69.

45. Sinclair AJ, Begg D, Mathai M, et al. (2007) Omega 3 fatty acids and the brain: review of studies in depression. Asia Pac J Clin Nutr 1, 391-397.

46. Meyer BJ, Grenyer BF, Crowe T, et al. (2013) Improvement of major depression is associated with increased erythrocyte DHA. Lipids 48, 863-886. 